\title{
Intense THz radiation produced in organic salt crystals for high-field applications
}

\author{
C. Vicario ${ }^{1}$, C. Ruchert ${ }^{1}$ and C. P. Hauri ${ }^{1,2}$ \\ 1Paul Scherrer Institute, 5232 Villigen PSI, Switzerland; \\ 2 Ecole Polytechnique Federale de Lausanne, 1015 Lausanne, Switzerland.
}

\begin{abstract}
Organic stilbazolium salt crystals pumped by intense, ultrashort mid-infrared laser have been investigated for efficient $\mathrm{THz}$ generation by optical rectification. In this paper we present our latest results in view of the generation of single-cycle and high-field $\mathrm{THz}$ transient in the THz gap (0.1-10 THz). The organic rectifiers like DAST, OH1 and DSTMS combine extremely large optical susceptibility with excellent velocity matching between the infrared pump and the $\mathrm{THz}$ radiation. Our simple collinear conversion scheme provides $\mathrm{THz}$ beams with excellent focusing properties and single cycle electric field larger than $1.5 \mathrm{MV} / \mathrm{cm}$ and magnetic field strength beyond 0.5 Tesla. The source can potentially cover the full THz gap at field strength which is barely provided by other THz sources. The $\mathrm{THz}$ pulse is carrier-envelope phase stable and the polarity of the field can be easily inverted.
\end{abstract}

\section{INTRODUCTION}

Intense coherent radiation in the Terahertz gap (0.1-10 THz) has been a challenge to produce in the past due to the lack of adequate gain materials in this spectral region. Terahertz radiation is suited to explore fundamental physical phenomena and to drive applications in condensed matter physics, biology, homeland security and medicine. Terahertz radiation is of growing interest since a series of resonant modes (magnons, phonons, electromagnons) are present in the THz gap, which provides a mean to control and investigate collective motions directly by THz pulses [1]. Furthermore, at increased field strength nonlinear dynamics are expected. The advent of $\mathrm{THz}$ pulses at high field strength is expected to permit new insight into collective effects in gases and solids, magnetization dynamics, and to realize charged

\footnotetext{
Nonlinear Frequency Generation and Conversion: Materials, Devices, and Applications XII edited by Konstantin L. Vodopyanov, Proc. of SPIE Vol. 8604, 86040C (c) 2013 SPIE - CCC code: $0277-786 X / 13 / \$ 18 \cdot$ doi: $10.1117 / 12.2010871$
} 
particle manipulation [2]. Moreover, high peak electric transients are required for the complete temporal reconstruction of ultrashort x-ray pulses generated in free electron lasers by a streak camera operating at THz frequencies [3].

While high field transient at frequencies between 20-100 THz (15 $\mu \mathrm{m}-3 \mu \mathrm{m})$, and thus outside the THz gap, has been recently demonstrated [4] the generation of high-power single-cycle field larger than a $\mathrm{MV} / \mathrm{cm}$ in the $\mathrm{THz}$ gap has remained challenging. Presently several schemes (based on laser or relativistic electron beam) are employed for the generation of such radiation [5,6]. Nevertheless, none of those fully satisfy the request of a reliable high- field few-cycle/single-cycle pulse with a spectrum spanning the full THz gap.

Recently a table-top source based on $\mathrm{LiNbO}_{3}$ demonstrated peak field of $1 \mathrm{MV} / \mathrm{cm}$ [6] and, with cryogenical cooling, pulse energies of up to $125 \mu \mathrm{J}$ could be realized [7], without field reconstruction though. The emitted spectra are centered at frequencies around $1 \mathrm{THz}$ and below.

To access significantly higher frequencies we recently developed a compact and powerful laser-driven $\mathrm{THz}$ source based on organic emitters. The radiation is generated by nonlinear optical rectification of femtosecond laser pulses in organic salt crystals like DAST [8], OH1 [9] and DSTMS [10]. The generation mechanism of THz radiation is understood as phase-matched nonlinear $\chi^{(2)}$ process. Different frequency components of the laser pulse with relative offset $\Delta \omega$ drive a nonlinear

polarization and radiation emission at frequency $\omega_{\mathrm{THz}}=\Delta \omega$ in the organic crystals. The broadband fundamental spectrum offers sufficient phase matching bandwidth to generate multi octave-spanning $\mathrm{THz}$ radiation. The difference frequency between components carrying the same absolute phase leads to intrinsically carrier phase stable pulses, which is essential for future field-sensitive experiments.

\section{EXPERIMENTAL SETUP}

The experimental setup is shown in figure 1 and is described in detail elsewhere [8]. In short, a TW-class Ti:Sa amplifier system at $100 \mathrm{~Hz}$ drives an optical parametric amplifier (OPA) and also provides a low energy probe pulse $(0.8 \mu \mathrm{m})$ for electro 
optical sampling (EOS) measurements. The powerful OPA produces pulses with 60 fs FWHM duration and energy up $3 \mathrm{~mJ}$ at wavelengths between 1.35 and $1.5 \mu \mathrm{m}$ and pumps the organic crystal in a collimated beam

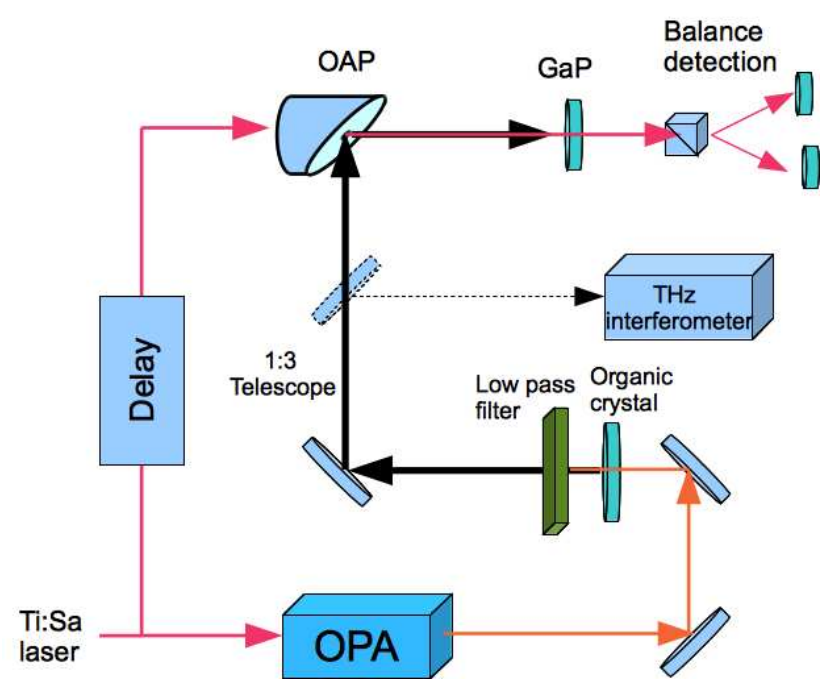

Figure 1. Experimental setup for the generation and the detection of the $\mathrm{THz}$ radiation. A Ti:Sa laser system is used to pump an optical parametric amplifier providing the right wavelength to drive optical rectification in organic crystal. Standard electro-optical sampling and interferometric measurement are employed for $\mathrm{THz}$ temporal and spectral characterization.

configuration. The $\mathrm{THz}$ is emitted collinearly to the pump with few-mrad divergence. Upstream the organic crystal, a thin sheet of Teflon or blackened Topas polymer is used as low pass filter to block the residual mid-IR beam. For reaching tightest spot size the $\mathrm{THz}$ beam is expanded and focused by an off axis parabolic (OAP) mirror ( $\mathrm{f}=101.6 \mathrm{~mm}$ ). The OAP mirror is equipped with a $3 \mathrm{~mm}$ central hole to recombine the $\mathrm{THz}$ and the probe beam for EOS. The THz pulse characteristics are provided by EOS giving direct access to the THz electric shape, peak intensity and to the spectral properties (via through Fourier transformation). In the electro-optical $\mathrm{GaP}$ crystal, the THz transient induces an instantaneous birefringence probed by the 
$800 \mathrm{~nm}$ laser pulse. In our experiment, the electro-optical spectral response and the velocity mismatch between $\mathrm{THz}$ and probe pulse limit the detectable spectrum in the GaP (95 $\mu \mathrm{m}$ thickness, (110) cut) to $7 \mathrm{THz}$. For measuring spectra carrying higher frequencies (up to $20 \mathrm{THz}$ ) we employed Fourier Transform linear interferometry by a Michelson interferometer equipped with THz-sensitive optics and detectors [11]. For absolute energy measurement a calibrated Golay cell and a pyroelectric detector are used. The transverse beam profile at the focus is recorded with a bolometer camera with pixel size of 23.5 microns.

\section{EXPERIMENTAL RESULTS}

\section{High field generation}

THz emission is performed in large size DAST, DSTMS and OH1 organic crystals. In order to accept the large pump laser energy the crystals are grown up to $10 \mathrm{~mm}$ apertures and carry a nominal thickness of $0.5 \mathrm{~mm}$. The optical rectification in those organic salt materials is very efficient and permits the realization of extremely large $\mathrm{THz}$ electro-magnetic fields. Organic salt crystals provide in fact low $\mathrm{THz}$ absorption and optical susceptibility $\chi^{(2)}$ one order of magnitude larger than uncooled $\mathrm{LiNbO}_{3}$ [7]. Velocity matching can be easily achieved at pump wavelengths between 1.35 and $1.5 \mu \mathrm{m}$.

The temporal evolution of the electric field for DAST, OH1 and DSTMS are shown in figure 2. High energy per pulse and very tight focus allow for the highest peak $\mathrm{THz}$ electric field. Transients in excess of $1 \mathrm{MV} / \mathrm{cm}$ are recorded for different organic crystals. The electric field is calibrated through the absolute rotation in the probe pulse polarization induced by the THz transient and cross-checked by measuring the THz pulse energy, duration and transverse spot size. The corresponding spectra are reported in the lower graphs. For all the crystals, the THz transient is characterized by single-cycle temporal oscillation with different grade of asymmetry. The difference in absolute phase originates from the $\mathrm{THz}$ beam propagation in the 
applied low pass filters and by the waist position where the EOS is performed (due to the Guoy effect). The generated THz transient is carrier envelope phase stable
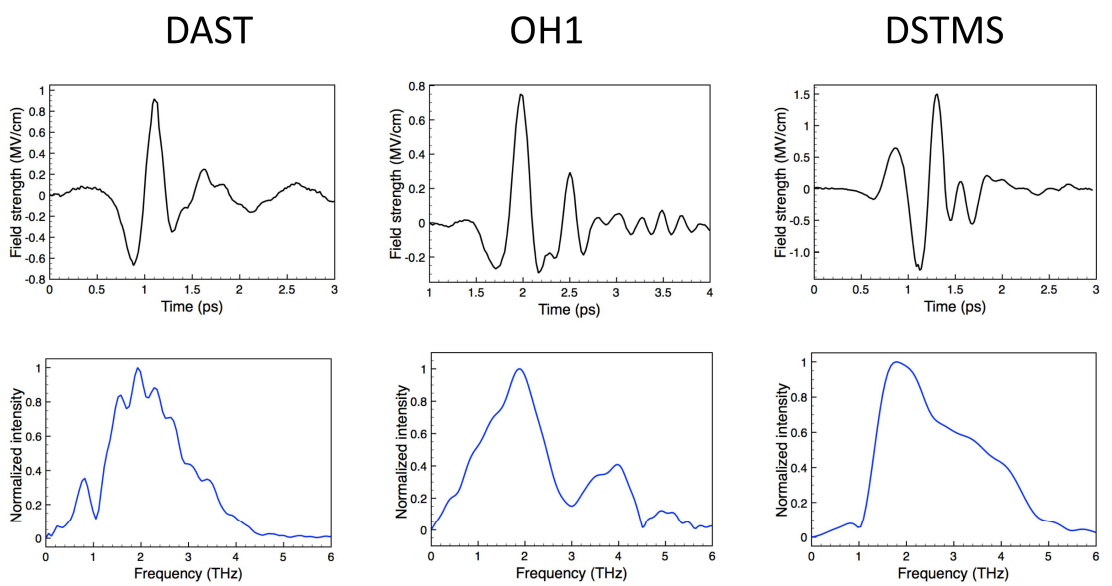

Figure 2. Temporal and spectral THz features for different organic materials. The optical rectification is driven by a high intensity pump (1.35-1.5 $\mu \mathrm{m})$ with a fluence of up to $160 \mathrm{GW} / \mathrm{cm}^{2}$.

with the possibility to invert the field polarity by simply rotation by 180 degree the emitter.

The maximum field recorded for the sample DSTMS B65 displays peak field in excess of $1.5 \mathrm{MV} / \mathrm{cm}$ and magnetic field of 0.5 Tesla. For OH1 and DAST organic

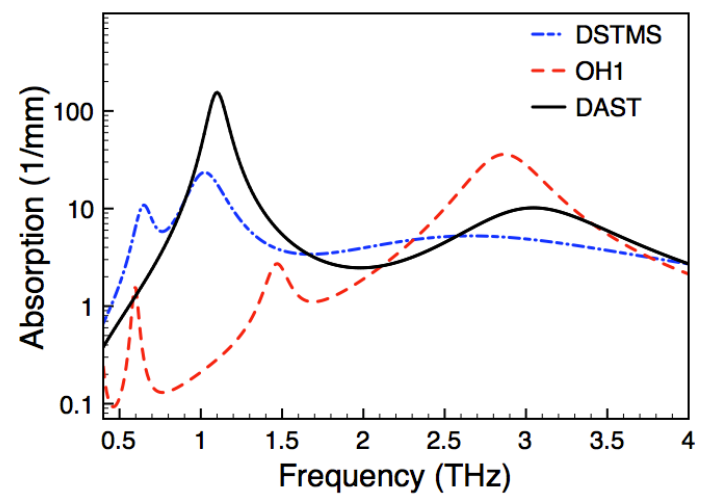

Figure 3. Theoretical absorption curves for DAST, DSTMS and OH1 between 0.4 and $4 \mathrm{THz}$. Phonon excitations induce strong absorption in the organic crystals at different frequencies. DAST is characterized by strong phonon absorption at $1 \mathrm{THz}$. 
OH1 show a relative low absorption and smooth spectrum for frequency below 3 THz. DSTMS presents absorption at 0.6 and $1 \mathrm{THz}$ and an average absorption lower than the other organic materials.

crystal the peak field is slightly less $(\approx 1 \mathrm{MV} / \mathrm{cm})$ when pumped by the same pump fluence. The corresponding spectra reveal the absorption properties and the phonon resonances of the different organic material. Due to these phonon resonances, displayed in figure 3, the temporal transients present post oscillations. The DAST spectrum, for example, is characterized by strong absorption at frequencies lower than $1 \mathrm{THz}$ and extends up to $4.5 \mathrm{THz}$. The $\mathrm{OH} 1$ emitted frequencies are located at frequencies smaller than $3 \mathrm{THz}$. The DSTMS produce radiation between 1 and $5 \mathrm{THz}$ without significant absorptions. The resonances and the absorption of the organic materials have been extensively characterized up to $4 \mathrm{THz}$ in Ref. [12]. To reduce Fresnel losses the organic crystals are coated with anti-reflection layer. The damage threshold for a femtosecond pump has been measured to be above $160 \mathrm{GW} / \mathrm{cm}^{2}$. A series of experimental data are summarized in table 1.

Table $1 \mathrm{THz}$ emission properties for DAST, DSTMS and OH1.

\begin{tabular}{|l|c|c|c|}
\hline & DAST & OH1 & DSTMS \\
\hline central frequency (THz) & 2.5 & 1.8 & 3 \\
\hline energy conversion efficiency $(\%)$ & 2 & 1.5 & 0.8 \\
\hline max THz energy $(\mu \mathrm{J})$ & 45 & 9 & 15 \\
\hline focal spot size $(\mathrm{mm})$ & 0.6 & 1.2 & 0.35 \\
\hline peak electric field $(\mathrm{MV} / \mathrm{cm})$ & 0.95 & 0.44 & 1.5 \\
\hline bandwidth $\Delta \mathrm{f}(\mathrm{THz})$ & 3.5 & 1.4 & 2 \\
\hline peak frequency $\mathrm{f}_{0}(\mathrm{THz})$ & 2.3 & 1.8 & 2.4 \\
\hline
\end{tabular}




\section{Pump fluence scaling}

Shown in figure 4 is the THz pulse energy generated in the DAST crystal as function of the infrared laser energy and laser intensity, respectively. The absolute $\mathrm{THz}$ energy is measured with a calibrated Golay cell positioned after the low pass filter

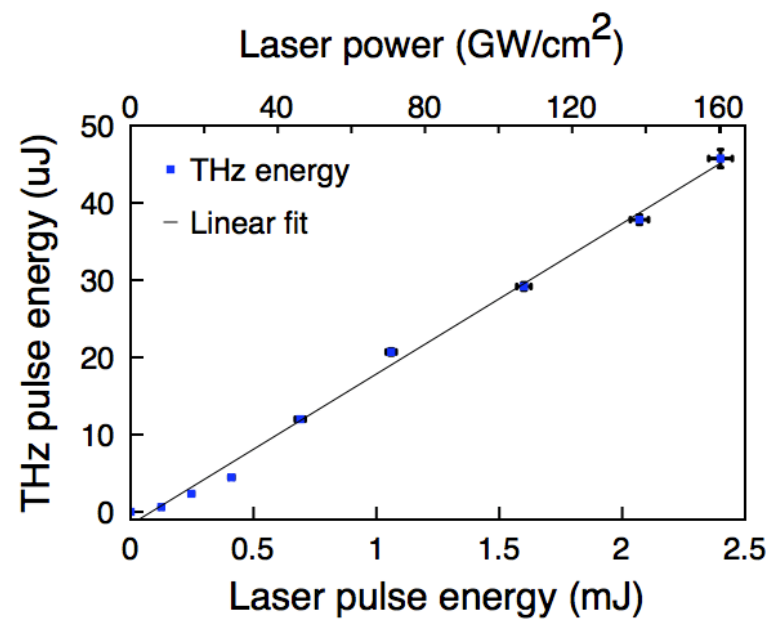

Figure 4. THz energy measured with a calibrated Golay cell, plotted as function of the infrared pump energy for DAST. Energy stability of the THz pulse is comparable to the pump energy stability and equal to $1 \% \mathrm{rms}$.

and calibrated $\mathrm{THz}$ attenuators. The maximum energy for $\mathrm{THz}$ pulse is larger than $45 \mu \mathrm{J}$ when the crystal is pumped by $3 \mathrm{~mJ}(160 \mathrm{GW} / \mathrm{cm} 2)$. The energy dependence is approximately linear and the pump-to-THz energy conversion is slightly less than $2 \%$ corresponding to a photon conversion efficiency of $200 \%$. The shot-to-shot stability of the $\mathrm{THz}$ energy is excellent. The energy variation recorded over 500 consecutive shots is $1 \% \mathrm{rms}$ and is equal to the stability of the OPA (horiz. error bars in figure 4). The linear dependence of the $\mathrm{THz}$ energy on the pump energy makes further upscaling feasible. It is worth noting maximum power density of 160 $\mathrm{GW} / \mathrm{cm}^{2}$ is applied without causing any damage of the organic crystal. For other organic material the $\mathrm{THz}$ energy yield recorded in the same experimental conditions is approximately $1 \%$ as reported in table 1 . 


\section{THz focus}

The $\mathrm{THz}$ and pump velocity matching allows collinear conversion geometry and aberration-free $\mathrm{THz}$ generation. The $\mathrm{THz}$ beam is produced over an extended crystal area of several square millimeters illuminated by the pump. The consequent divergence of the $\mathrm{THz}$ beam is only a few mrad. In our setup a tight focal spot is reached when the beam is expanded by factor 3 prior to focusing by $10 \mathrm{~cm}$ OAP. In figure 5, we present a typical THz spot size. The profile is recorded with uncooled bolometer array sensor with pixel pitch of $23.5 \mu \mathrm{m}$. The transverse beam shape at the focus is circular and not affected by astigmatism. As shown in the projected profile the intensity is well approximated by a Gaussian curve with full width half maximum of $360 \mu \mathrm{m}$ close to the diffraction limit. The beam size measurement carried out for other crystals indicate a beam size of sub-mm and absence of significant aberrations (see table 1).

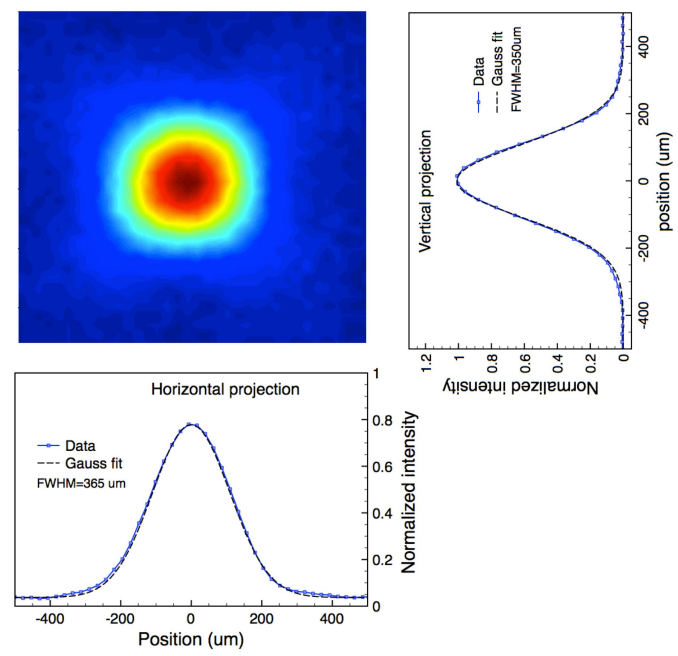

Figure 5. THz radiation generated in organic crystal can be focused very tightly. The focused beam generated in DSTMS is shown together with its vertical and horizontal projection. The beam is recorded with a micro-bolometer camera with 23.5 microns pitch size. The spot is well approximated by a Gaussian function with a FWHM of 350 and $366 \mu \mathrm{m}$ in vertical and horizontal projection. 


\section{Ultra-broadband THz generation}

Both the phase velocity mismatch and the $\mathrm{THz}$ re-absorption define the optimal thickness of the organic crystal and subsequently the generated $\mathrm{THz}$ spectral content. For $\mathrm{THz}$ generation at higher frequencies thinner crystals are required. In our investigations the largest $\mathrm{THz}$ spectrum is generated in a thin $\mathrm{OH} 1$ crystal of 180 $\mu \mathrm{m}$ thickness with the results shown in figure 7. To avoid limitation in the electrooptic material, the EOS measurement is replaced by Fourier interferometry. Furthermore the low pass Teflon filter is replaced by a carbon-doped Topas polymer, which is characterized by low absorption for the entire THz gap. In figure 7 the interferometric trace and the corresponding spectrum are shown. The generated spectrum is impressively large and covers the full range of the THz gap up to 20 THz.
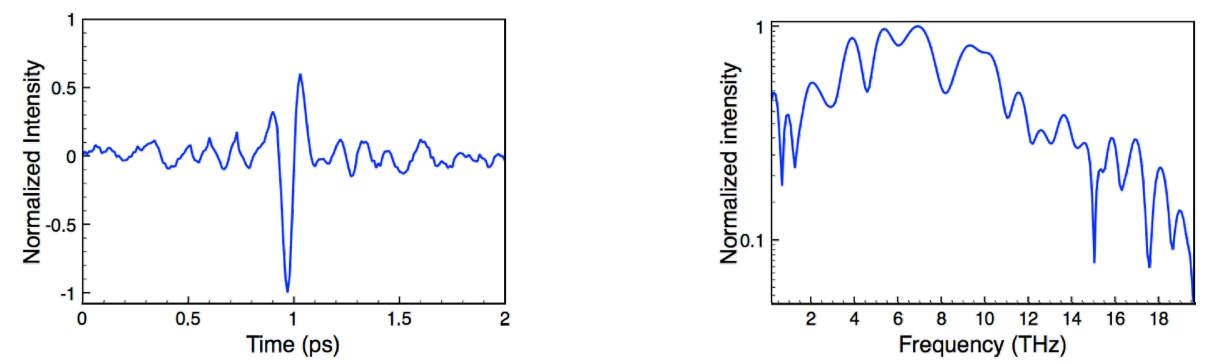

Figure 6. Interferometric measurement and reconstructed spectrum generated in 180 um-thick $\mathrm{OH} 1$ crystal. The generated spectrum covers the entire THz gap. Several absorption peaks due to phonon resonances in the crystal are visible. 


\section{CONCLUSIONS}

In this paper we investigated a series of organic crystals (OH1, DAST, DSTMS) in view of their potential for high-field THz generation in the THz gap (0.1-10 THz). All crystals turned out to be highly efficient (up to 2\%) and broadband IR-to-THz converters when pumped by a mJ femtosecond mid-infrared pulse. The generated $\mathrm{THz}$ radiation offers multi-octave spanning, single-cycle and phase-stable $\mathrm{THz}$ pulses with up to $1.5 \mathrm{MV} / \mathrm{cm}$ electric field strength. The generation scheme based on a collimated pumping geometry provides excellent $\mathrm{THz}$ focusing characteristics. The THz spectra show modulations which are caused by the crystal-related phonon absorptions. Ultra-broadband $\mathrm{THz}$ spectra covering the frequency range of $1-20 \mathrm{THz}$ could be realized by optical rectification in a thin organic crystal. The THz source presented here will be of benefit for field-sensitive investigations to drive extreme nonlinear phenomena in gases and solids with single-cycle transients.

\section{ACKNOWLEDGEMENTS}

We acknowledge support from Swiss National Science Foundation (grant no. PP00P2128493 and 20002-1122111) and SwissFEL.

\section{BIBLIOGRAPHY}

[1] M. Tonouchi, “Cutting-edge terahertz technology," Nature Photon. 1, 97 (2007).

[2] E. Balogh, K. Kovacs, P. Dombi, J. A. Fulop, G. Farkas, J Hebling, V Tosa, and K. Varju "Single attosecond pulse from terahertz-assisted high-order harmonic generation," Phys. Rev. A 84, 023806 (2011).

[3] U. Fruehling, M. Wieland, M. Gensch, T. Gebert, B. Schuette, M. Krikunova, R. Kalms, F. Budzyn, O. Grimm, J. Rossbach, E. Ploenjes, M. Drescher, "Single-shot terahertz-field-driven X-ray streak camera," Nature Photon. 3, 523 (2009).

[4] F. Jungiger, A. Sell, O. Schubert, B. Mayer, D. Brida, M. Marangoni, G. Cerullo, A. Leitenstorfer, R. Huber, "Single-cycle multiterahertz transients with peak fields above $10 \mathrm{MV} / \mathrm{cm}$," Opt. Lett. 35, 2645 (2010). 
[5] D. Daranciang, J. Goodfellow, M. Fuchs, H. Wen, S. Ghimire, D. A. Reis, H. Loos, A. S. Fisher and A. M. Lindenberg, "Single-cycle terahertz pulses with $>0.2 \mathrm{~V} / \mathrm{A}$ field amplitudes via coherent transition radiation,“ Appl. Phys. Lett. 99, 141117 (2011).

[6] H. Hirori, A. Doi, F. Blanchard, K. Tanaka, "Single-cycle terahertz pulses with amplitudes exceeding $1 \mathrm{MV} / \mathrm{cm}$ generated by optical rectification in $\mathrm{LiNbO}_{3}, "$ Appl. Phys. Lett. 98, 091106 (2011).

[7] J. A. Fülöp, L. Pálfalvi, S. Klingebiel, G. Almási, F. Krausz, S. Karsch and J. Hebling "Generation of sub-mJ terahertz pulses by optical rectification," Opt. Lett. 37, 4, 557 (2012).

[8] C. P. Hauri, C. Ruchert, F. Ardana and C. Vicario "Strong-field single-cycle THz pulse generated in organic crystal," Appl. Phys. Lett. 99, 161116 (2011).

[9] C. Ruchert, C. Vicario and C. P. Hauri, "Scaling Sub-mm single-cycle transients towards $\mathrm{MV} / \mathrm{cm}$ fields via optical rectification in organic crystal $\mathrm{OH} 1$," Opt. Lett. 37, 899 (2012).

[10] C. Ruchert, C. Vicario and C. P. Hauri, Spatio-temporal focusing dynamics of intense supercontinuum THz pulses," Phys. Rev. Lett. (submitted)

[11] www.radiabeam.com

[12] L. Mutter, F. D. Brunner, Z. Yang, M. Jazbinsek, and P. Günter, "Linear and nonlinear optical properties of the organic crystal dstms," J. Opt. Soc. Am. B 24, 2556 (2007). 